\title{
Noise induced entanglement
}

\author{
J. Chen, X. X. Yi *, H. S. Song, and L. Zhou \\ Department of physics, Dalian University of Technology, Dalian 116024, China
}

(Dated: November 9, 2018)

\begin{abstract}
We discuss the generation of entangled states of two two-level atoms coupled simultaneously with a dissipated atom. The dissipation of the atom is supposed to come from its coupling to a noise with adjustable intensity. We describe how the entanglement between the atoms arise in such a situation, and wether a noise except the white one could help preparation of entanglement. Besides, we confirm that the entanglement is maximized for intermediate values of the noise intensity, while it is a monotonic function of the spontaneous rates. This resembles the phenomenon of stochastic resonance and sheds more light on the idea to exploit noise in quantum information processing.
\end{abstract}

PACS numbers: 03.67.-a, 03.67.-Hz

Entanglement lies at the heart of quantum information processing (QIP) [1, 2, 3], preparation of entanglement as a physical resource is thus a primary goal of this field. Entangled two two-level atoms provide an ideal model for quantum teleportation as well as a simple description for theoretical studies in QIP, this makes the preparation of entangled two two-level atoms attractive and interesting. The main problem that must be overcome in QIP is decoherence, an effect that results from the coupling of the system to its surroundings or from the inability to control precisely experimental parameters. A consequence of decoherence is that the entangled system may end up in a mixed state that would be no longer useful for any quantum information processing. It is therefore important for practical realization of quantum information processing protocols to engineer mechanisms to prevent, minimize, or use the impact of environmental noise.

There are a lot of proposals that have been made for preventing, minimizing or using the environmental noise, for example, loop control strategies, that use an ancillary system coupling to the quantum processor to better the performance of the proposals [4, 5], quantum error correction [6] uses redundant coding to protect quantum states against noisy environments. This procedure is successful as long as the error rate is sufficiently small. It wastes a number of qubits and quantum gates, and then limit its implementation by present available technology. A more economic approach consists of exploiting the existence of so-called decoherence-free subspace that are completely insensitive to specific types of noise [7]. This approach tends to require fewer additional resources, but is only applicable in specific situations. The seminal idea that dissipation can assist the generation of entanglement has been put forward recently $[8,9,10]$. In a system consisting of two distinct leaky optical cavities, it was shown that the entanglement is maximized for intermediate values of the cavity damping rates and the intensity of the

\footnotetext{
${ }^{*}$ Corresponding author
}

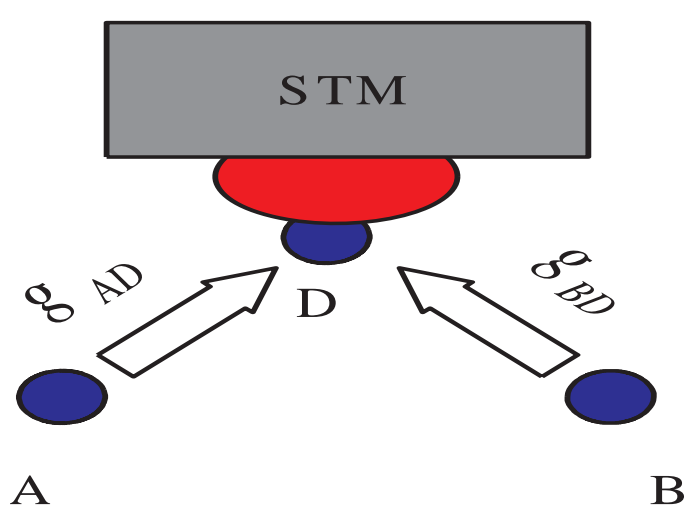

FIG. 1: Envisaged setup for the preparation of two entangled atoms $A$ and $B$. The atom $D$ (Detector) is placed at the STM tip, it couples to the tip of the STM and then is dissipated. The three atoms are initially prepared in their ground state $|g\rangle_{A}|g\rangle_{B}|g\rangle_{D}$. The atoms $A$ and $B$ become entangled and the entanglement is maximized for intermediate values of the noise intensity.

white noise, vanishing both for small and for large values of these parameters [10]. In fact, this idea appeared first in Ref. [8] for two atoms inside an optical cavity and it shows that cavity decay can assist the preparation of maximally entangled atoms, without cavity decay, the reduced state of the two-atom system would be in an inseparable mixture at all times, but not in a maximally entangled one.

In a recent paper, the idea in Ref. 10] has been put forward to a two-atom system [1], the author used white noise to play a constructive role in the entanglement preparation, and shown numerically that controllable entanglement may arise indeed in that situation. There are two questions arise naturally, (1) if the other kind of noise except the white one can assist preparation of entanglement and (2) how the systems are entangled with assistance of noise. The main goal of this paper is to answer these questions.

Our system consists of three two-level atoms as depicted in figure 1 , we will refer to atom $A$, atom $B$ 
and atom $D$ when the context requires us to differentiate them, but otherwise they are supposed to be identical. We denote the atomic ground and excited states by $|g\rangle_{i}$ and $|e\rangle_{i}$, respectively, and call $2 \gamma_{i}(i=A, B, D)$ the spontaneous emission rate from the upper level. We will refer $\sigma_{i}^{+}=|e\rangle_{i}\langle g|$ to the pauli operator for atom $i$. The atom $D$ is driven by a noise whose intensity will be characterized by the effective particle number $n_{T}$. In fact the tip connected the atom $D$ may play the role of the noise. The master equation governing the time evolution of the global system is given by (setting $\hbar=1$ )

$$
\dot{\rho}=-i[H, \rho]+\mathcal{L}(\rho),
$$

where the Hamiltonian $H$ describes the internal energies of the atoms as well as the inter-atom couplings. The Liouvillean $\mathcal{L}(\rho)$ describes the decay of atoms $A$ and $B$, as well as the interaction of the atom $D$ with the noise. As no external coherent driving is present, the Hamiltonian reads

$$
H=\sum_{i=A, B, D} \frac{\omega_{i}}{2} \sigma_{i}^{z}+\sum_{i=A, B} g_{i D}\left(\sigma_{D}^{+} \sigma_{i}^{-}+\text {h.c. }\right),
$$

when the noise is a white one, the Liouvillean is given by 12.

$$
\begin{aligned}
\mathcal{L}(\rho) & =-\gamma_{D}\left(n_{T}+1\right)\left(\sigma_{D}^{+} \sigma_{D}^{-} \rho+\rho \sigma_{D}^{+} \sigma_{D}^{-}-2 \sigma_{D}^{-} \rho \sigma_{D}^{+}\right) \\
& -\gamma_{D} n_{T}\left(\sigma_{D}^{-} \sigma_{D}^{+} \rho+\rho \sigma_{D}^{-} \sigma_{D}^{+}-2 \sigma_{D}^{+} \rho \sigma_{D}^{-}\right) \\
& -\sum_{i=A, B} \gamma_{i}\left(|e\rangle_{i}\langle e|\rho+\rho| e\rangle_{i}\langle e|-2| g\rangle_{i}\langle e|\rho| e\rangle_{i}\langle g|\right)
\end{aligned}
$$

Here $\gamma_{i}(i=A, B)$ describes the atom decay rate and we assume $\gamma_{A}=\gamma_{B}=\gamma, n_{T}$ stands for the intensity of the white noise, which refers to its effective particle number. To simplify the representation, now we turn to an interaction picture with respect to $H_{0}=\sum_{i=A, B, D} \frac{\omega}{2} \sigma_{i}^{z}$. After this transformation, the Liouvillean part remains unchanged, while the Hamiltonian part is now given by

$$
H_{I}=\sum_{i=A, B} g_{i D}\left(\sigma_{D}^{+} \sigma_{i}^{-}+h . c .\right)
$$

where we assume that the three atoms are identical, i.e., with the same free Rabi frequency $\omega_{i}=\omega$. The analytical solution to the equation (1) is extremely tedious. We will now present numerical simulations to show how the entanglement in system $A$ and $B$ depend on the noise intensity, time $t$ and the decay rate of atoms $A$ and $B$.

We will choose the Wootters concurrence as the entanglement measure 13],

$$
c(\rho)=\max \left\{0, \lambda_{1}-\lambda_{2}-\lambda_{3}-\lambda_{4}\right\},
$$

where the $\lambda_{i}$ are the square roots of the eigenvalues of the non-Hermitian matrix $\rho \tilde{\rho}$ with $\tilde{\rho}=\left(\sigma_{y} \otimes \sigma_{y}\right) \rho^{*}\left(\sigma_{y} \otimes \sigma_{y}\right)$
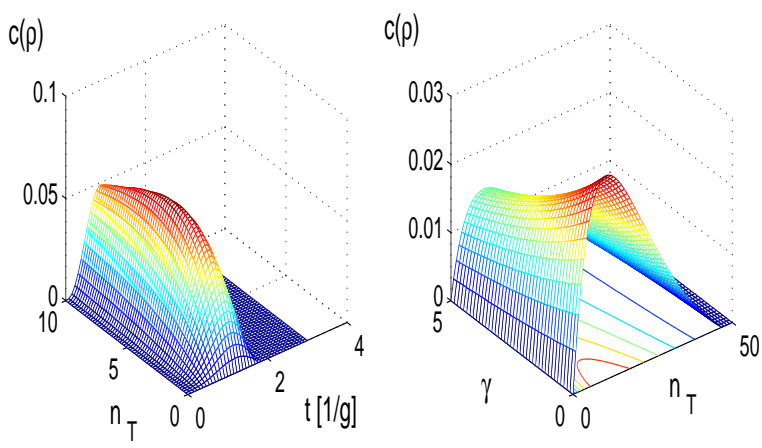

FIG. 2: left- Wootters concurence of the two-atom system as a function of the intensity of the white noise $n_{T}$ and time $t$. The chosen parameters are $g_{A D}=g_{B D}=1$ and $\gamma=\gamma_{D}=$ $0.2 g$. right- Wootters concurence as a function of the decay rate $\gamma$ (namely, the decay rate for atom $A$ and $B$ ) and the noise intensity $n_{T}$ with a specific time $t=1 / g$ and $\gamma_{D}=0.2 g$. The other parameters are the same as the left. Note that the entanglement arrives at its maximum for an intermediate noise intensity in both cases.

in decreasing order. The Wootters concurrence gives an explicit expression for the entanglement of formation, it quantifies the resources needed to create a given entangled state. The typical behavior of the entanglement in the system is illustrated in figure 2 . There we have plotted the amount of entanglement of the joint state of the two atoms as a two-variable function of the intensity of the noise $n_{T}$ and time $t$ (see the left panel of figure 2). We want to stress that our simulation is presented for Eq.(1), i.e., the original master equation for the three-atom system, and as we mentioned above the initial state of the global system is $|g\rangle_{A}|g\rangle_{B}|g\rangle_{D}$ in our simulation. Note that for any value of $t$ falling in the region of entanglement $\neq 0$, the behavior of the amount of entanglement between the two atoms is non-monotonic, it increases to a maximum value for an optimal intensity of the noise and then decreases towards zero for a sufficiently large intensity. Physically, to get non-zero amount of entanglement, the excited state of atom $D$ (act as a data bus here) must be populated at least one time. For the two limiting case of either $n_{T}=0$ or $n_{T} \rightarrow \infty$, however, the data bus remains idle for all the times. Thus the amount of entanglement equals zero. It is also worthwhile to study the dependence of entanglement on both the intensity of the noise and the atom decay rates $\gamma$ of $A$ and $B$. In the right panel of figure 2 , we present those dependence of the entanglement on $\gamma$ and $n_{T}$. It is interesting to note that the amount of entanglement behave as a monotonic function of the atom decay rate $\gamma$. This is quite different from the case presented in Ref. [10], where the cavity decay can assist themselves to prepare entanglement shared among them.

Now we turn to study the case where the noise is a 

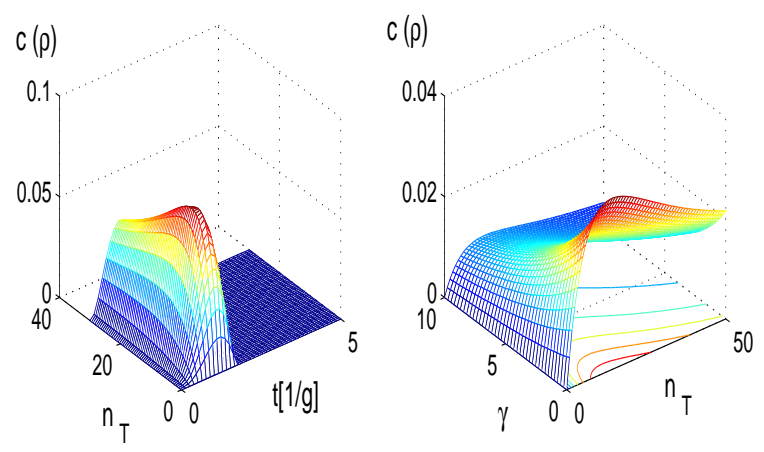

FIG. 3: The same as figure 2, but for the squeezing noise.

squeezed white noise. To describe the dynamical evolution of the system under influence of the squeezed white noise, terms

$$
-\gamma_{D} M\left(\sigma_{D}^{-} \sigma_{D}^{-} \rho+\rho \sigma_{D}^{-} \sigma_{D}^{-}-2 \sigma_{D}^{-} \rho \sigma_{D}^{-}\right)+\text {h.c. },
$$

should be added to the Liouvillean Eq.(3). Where $M$ together with $n_{T}$ characterize the noise and fulfill $M \leq$ $\sqrt{n_{T}\left(n_{T}+1\right)}$. Here we will consider the ideal case in which $M=\sqrt{n_{T}\left(n_{T}+1\right)}$, i.e., the perfect squeezing case. The numerical results for this situation were presented in figure 3. By comparing figure 2 with figure 3 we find that the entanglement are maximized for different values of $n_{T}$ in the two case.

To shed more light on the entanglement preparation, we now show in a analytical way how the entanglements are generated in the system. To make the physics clear, we introduce two new effective atomic modes and choose the case of white noise as a demonstration. As we will see, one of the effective modes will be decoupled from the system. The two collective atomic modes are given by the following definition

$$
\sigma_{C}^{+}=\frac{g_{A D} \sigma_{A}^{+}+g_{B D} \sigma_{B}^{+}}{\sqrt{g_{A D}^{2}+g_{B D}^{2}}}, \sigma_{E}^{+}=\frac{g_{B D} \sigma_{A}^{+}-g_{A D} \sigma_{B}^{+}}{\sqrt{g_{A D}^{2}+g_{B D}^{2}}},
$$

and $\sigma_{i}^{-}=\left(\sigma_{i}^{+}\right)^{\dagger}$. In terms of these new operators, the Hamiltonian and Liouvillean part of the master equation are given by

$$
H_{I}=g_{C D}\left(\sigma_{D}^{+} \sigma_{C}^{-}+\text {h.c. }\right),
$$

where $g=\sqrt{g_{A D}^{2}+g_{B D}^{2}}$, and

$$
\mathcal{L}(\rho)=-\gamma_{D}\left(n_{T}+1\right)\left(\sigma_{D}^{+} \sigma_{D}^{-} \rho+\rho \sigma_{D}^{+} \sigma_{D}^{-}-2 \sigma_{D}^{-} \rho \sigma_{D}^{+}\right)
$$

$$
\begin{aligned}
& -\gamma_{D} n_{T}\left(\sigma_{D}^{-} \sigma_{D}^{+} \rho+\rho \sigma_{D}^{-} \sigma_{D}^{+}-2 \sigma_{D}^{+} \rho \sigma_{D}^{-}\right) \\
& -\gamma \sum_{i=C, E}\left(|e\rangle_{i}\langle e|\rho+\rho| e\rangle_{i}\langle e|-2| g\rangle_{i}\langle e|\rho| e\rangle_{i}\langle g|\right) .
\end{aligned}
$$

Note that the sum in the last line of Eq.(7) is taken over the two NEW modes. The transformation between the resulting atom $A, B$ and the collective modes $C, E$ is clear. For example, both the resulting atoms $A$ and $B$ in its ground state $|g\rangle_{A}|g\rangle_{B}$ can be equivalently expressed in terms of $|g\rangle_{C}|g\rangle_{E}$ and $|e\rangle_{A}|e\rangle_{B}$ likewise. The new master equation Eq. (7) shows us that we have one mode (mode $E$ ) which is completely decoupled from the Hamiltonian dynamics and is purely damped under the Liouvillean dynamics, this is a consequence of the transformation from the resulting atoms to the collective modes. The mode $E$ then will not be populated in steady state irrespective of its initial states. In other words, if the mode $E$ is in its ground state initially, it will remain on that forever. Therefore, we begin our investigations with both collective modes $C$ and $E$ in the ground state $|g\rangle_{C}|g\rangle_{E}$. As the mode $E$ will then never be populated, we disregard that mode in the following discussions. Apart from the above assumption, we discuss the entanglement generation here only for the case of no initial population on the excited state of atom $D$, this is relevant to the topics under our consideration, i.e., study the role of the white noise in the entanglement generation. To understand the origin of the generation of entanglement from the white noise, let us first show how the two modes $C$ and $D$ are entangled. To this end, we solve perturbatively the master equation for modes $C$ and $D$ by expanding the density matrix $\rho_{C D}(t)$ as a power of short time $t$, it yields

$$
\rho_{C D}(t)=\rho_{C D}(0)+\rho_{C D}^{(1)} t+\frac{1}{2 !} \rho_{C D}^{(2)} t^{2}+\ldots
$$

This expansion is a good approach to the solution of the master equation with Hamiltonian Eq.(7) and Liouvillean Eq.(8) in the limit $t \rightarrow 0$. Eq. (9) and Eqs $(1,7,8)$ together give

$$
\begin{aligned}
& \rho_{C D}^{(1)}=-i\left[H, \rho_{C D}(0)\right]+\mathcal{L}\left(\rho_{C D}(0)\right), \\
& \rho_{C D}^{(2)}=-i\left[H, \rho_{C D}^{(1)}\right]+\mathcal{L}\left(\rho_{C D}^{(1)}\right),
\end{aligned}
$$

For a specific initial state $\rho_{C D}(0)=|g g\rangle\langle g g|$, to the second order of time, the density matrix $\rho_{C D}(t)$ in the basis $\{|g g\rangle,|g e\rangle,|e g\rangle,|e e\rangle\}$ reads 


$$
\rho_{C D}(t)=\left(\begin{array}{cccc}
1-\gamma_{D} n_{T} t+\frac{\gamma_{D}^{2} n_{T}\left(n_{T}+1\right)+\gamma_{D}^{2} n_{T}^{2}}{2} t^{2} & 0 & 0 & 0 \\
0 & \gamma_{D} n_{T} t-\frac{\gamma_{D}^{2} n_{T}\left(n_{T}+1\right)+\gamma_{D}^{2} n_{T}^{2}}{2} t^{2} & -\frac{i g \gamma_{D} n_{T}}{2} t^{2} & 0 \\
0 & \frac{i g \gamma_{D} n_{T}}{2} t^{2} & 0 & 0 \\
0 & 0 & 0 & 0
\end{array}\right) .
$$

The Wootters concurence for this state is $g \gamma_{D} n_{T} t^{2}$. The physics of this result is clear, to get nonzero entanglement in modes $C$ and $D$, either the coupling constant $g$, the decay rate $\gamma_{D}$ or the noise intensity $n_{T}$ could not be zero, and the entanglement increase linearly with $g$, $\gamma_{D}$ and $n_{T}$ at the beginning of evolution. It is worthy to stress that the entanglement shared between $C$ and $D$ do not indicate certainly that the resulting modes $A$ and $B$ are entangled. In fact, it is easy to check that $A$ and $B$ are in a separable state up to the any order of $t$. They would not be entangled within the short-time approximation. Although the resulting modes $A$ and $B$ could not end up in an entangled state within the short time approximation, the short time approach provided us a manner of how two modes become entangled starting from their ground state via coupling to a noise. The entanglement share among $A$ and $B$ arises exactly in the same manner.

The noise-assisted entanglement preparation is somehow reminiscent of the well known phenomenon of stochastic resonance [14, 15, 16], where the response of a system to a periodic force can be enhanced in the presence of an intermediate amount of noise. A related effect that cavity decay can assist the generation of squeezing has been found recently [17], there they shown that the squeezing effect is enhanced as the damping rate of the cavity is increased to some extent, and the pumping field amplitude is required to be inversely proportional to the damping rate for the optimal squeezing.

As an example, we now describe a setup for entangled atom pair creation with two ${ }^{31} \mathrm{P}$ ions deposited at the (111) surface of ${ }^{28} \mathrm{Si}$ substrate. As the author did in Ref. [18], we choose ${ }^{31} P$ that has nuclear spin $I=\frac{1}{2}$ act as the two-level system. $\mathrm{A}^{13} \mathrm{C}$ was put on the tip of STM, the ${ }^{31} P$ ions in the substrate was deposited with a large distance such that no direct interaction between them. With the STM tip approaches the two ${ }^{31} P$ ions, the entanglement starts to be generated and the two ${ }^{31} P$ ions might end up in an entangled state.

To sum up, we have described an experimental situation where entanglement between two atomic systems can be prepared with assistance of a noise, the noise might be a white one or a squeezed white one. The entanglement measured by the Wootters concurence is maximized for intermediate values of the intensity of the noise, while it is a monotonic function of the atomic spontaneous emission rate. Recall that the atomic decay itself can not induce entanglement among the atoms, even if at finite temperatures, we conclude that the coupling between the data bus and the white noise is the origin of the generation of the entanglement. The phenomenon of white noise-assisted entanglement generation is not a rare phenomenon, it resembles the phenomenon of stochastic resonance. However, this discovery [10] is really valuable because it sheds new light on the constructive role that noise may play in quantum information processing. In contrast with the results in Ref. 10], the proposal presented here is for the entanglement generation between two two-level atoms. For such a two-qubit system, any amount of entanglement, even if very small, is distillable 19], and therefore the entangled atoms are useful for quantum information processing.

This work is supported by EYTP of M.O.E, and NSF of China.

[1] C. H. Bennett and D. P. DiVincenzo, Nature 404, 247(2000).

[2] M. A. Nielsen and I. L. Chuang, Quantum computation and quantum information (Cambridge University press, Cambridge, 2000).

[3] M. B. Plenio and V. Vedral, Contemp. Phys. 39,431(1998).

[4] H. M. Wiseman and G. J. Milburn, Phys. Rev. Lett. 70,548(1993); D. Vital, et al., Phys. Rev. Lett.79, 2442(1997); L. Viola,et al., Phys. Rev. Lett. 82, 2417(1999).

[5] S. Mancini, et al., EuroPhys. Lett. 60, 498(2002).

[6] P. W. Shor, Phys. Rev. A 52, 2493(1995); A. R. Calderbank and P. W. Shor, Phys. Rev. A 54, 1098(1996); A. M. Steane, Proc. Roy. Soc. A 452, 2551(1996).

[7] G. M. Palma, et al., Proc. Roy. Soc. A 452, 567(1996); M. B. Plenio, et al., Phys. Rev. A 55, 67(1997); D. A. Lidar, et al., Phys. Rev. Lett. 81, 2594(1998); A. Beige, et al., Phys. Rev. Lett. 85, 1762(2000); A. Beige, et al., New J. Phys. 2, 22(2000).

[8] M. B. Plenio, et al., Phys. Rev. A 59, 2468(1999); A. Beige, et al., J. Mod. Opt. 47, 2583(2000); P. Horodecki, Phys. Rev. A 63,022108(2001).

[9] S. Bose, et al.,Phys. Rev. Lett. 83, 5158(1999); D. Braun, Phys. Rev. Lett. 89, 277901(2002).

[10] M. B. Plenio, et al., Phys. Rev. Lett. 88, 197901(2002). 
[11] X. X. Yi, et al., quant-ph/0306091 to appear in PRA.

[12] C. W. Gardiner and P. Zoller, Quantum Noise (Springer 2000).

[13] W. K. Wootters, Phys. Rev. Lett. 80, 2245(1998).

[14] L. Gammaitoni, et al., Rev. Mod. Phys. 70, 223(1998).

[15] S. F. Huelga and M. B. Plenio, Phys. Rev. A 62, 052111(2000).
[16] A. Buchleitner, and R. N. Mantegna, Phys. Rev. Lett.80, 3932(1998).

[17] H. Nha, et al., Phys. Rev. A 67, 023801(2003).

[18] G. L. Long et al., quant-ph/0307054

[19] M. Horodecki, et al., Phys. Rev. A 59, 4206 (1999); X. X. Yi, et al., Phys. Rev. A 62, 062312(2000). 